\title{
Cardiac pacing for severe childhood neurally mediated syncope with reflex anoxic seizures
}

\author{
K A McLeod, N Wilson, J Hewitt, J Norrie, J B P Stephenson
}

\begin{abstract}
Objective-To determine whether permanent cardiac pacing could prevent syncope and seizures in children with frequent severe neurally mediated syncope, and if so whether dual chamber pacing was superior to single chamber ventricular pacing.
\end{abstract}

Methods-Dual chamber pacemakers were implanted into 12 children (eight male, four female) aged 2-14 years (median 2.8 years) with frequent episodes of reflex anoxic seizures and a recorded prolonged asystole during an attack. The pacemaker was programmed to sensing only (ODO), single chamber ventricular pacing with hysteresis (VVI), and dual chamber pacing with rate drop response (DDD) for four month periods, with each patient allocated to one of the six possible sequences of these modes, according to chronological order of pacemaker implantation. The parent and patient were blinded to the pacemaker mode and asked to record all episodes of syncope or presyncope ("near miss" events). The doctor analysing the results was blinded to the patient and pacemaker mode.

Results-One patient was withdrawn from the study after the pacemaker was removed because of infection. In the remaining children, both dual chamber and single chamber pacing significantly reduced the number of syncopal episodes compared with sensing only $(p=0.0078$ for both). VVI was as effective as DDD for preventing syncope, but DDD was superior to VVI in reducing near miss events $(\mathrm{p}=0.016)$.

Conclusions-Permanent pacing is an effective treatment for children with severe neurally mediated syncope and reflex anoxic seizures. VVI is as effective as DDD in preventing syncope and seizures, but DDD is superior in preventing overall symptoms.

(Heart 1999;82:721-725)

Keywords: syncope; reflex anoxic seizures; pacing; paediatric cardiology

Neurally mediated syncopal attacks in childhood that are believed to be secondary to cardiac asystole have been variously described as pallid breath holding spells, ${ }^{12}$ white breath holding attacks, ${ }^{3}$ pallid infantile syncope, ${ }^{4}$ nonepileptic vagal attacks, ${ }^{5}$ and reflex anoxic seizures. ${ }^{5}{ }^{6}$ We use the term "reflex anoxic seizures" to describe the non-epileptic motor seizure characterised by stiffenings and jerks which accompanies syncope secondary to cardiac asystole in response to a noxious stimulus or unpleasant circumstance.

Reflex anoxic seizures are often dramatic and terrifying for patients and frequent seizures may seriously disrupt the lives of parents and child. They can precipitate true epileptic seizures $^{78}$ and even status epilepticus. ${ }^{9}$

Success in preventing reflex anoxic seizures through blockade of the vagus nerve by atropine or scopolamine has been reported, but these drugs are often not well tolerated. ${ }^{10}{ }^{11}$ Theoretically a cardiac pacemaker might prevent syncope and seizures by preventing asystole. There are a few reports of cessation of reflex anoxic seizures after cardiac pacing, but the studies involved very small numbers of patients and the placebo effect of pacing was not adequately tested. ${ }^{12-14}$ In adults, the role of pacemakers in the treatment of vasovagal syndromes has been controversial. ${ }^{15}{ }^{16}$ Not all patients benefit from pacing. Dual chamber pacing appears to be superior to ventricular pacing, and dual chamber pacing with the rate drop response may be most effective. ${ }^{17} 18$

In the present study we aimed to determine, in a double blind trial, whether reflex anoxic seizures can be prevented by cardiac pacing, and if so, whether dual chamber pacing with rate drop response was superior to single chamber ventricular pacing.

\section{Methods}

Children were eligible for the study if they were over 2 years old, had a history typical of reflex anoxic seizures (as outlined above), had a cardiac asystole of $>4$ seconds recorded during a typical attack with loss of consciousness (fig 1), and had reflex anoxic seizures at least once a week. Although frequent severe reflex anoxic seizures are more common in younger children, children under the age of 2 years were excluded from the studies owing to concerns about the implantation of dual chamber systems in very small children. In children of 5 years or less, asystole was recorded on a cardiac event monitor during a spontaneous attack; in children over 6 years old, it was recorded during tilt testing.

The Thera DR small (Medtronic, Minneapolis, USA) pacemaker was chosen for the study because it is the smallest dual chamber pacemaker available with the rate drop response. Bipolar $45 \mathrm{~cm}(53 \mathrm{~cm}$ in the eldest patient), $7 \mathrm{~F}$ tined preshaped atrial $\mathrm{J}$ leads (Medtronic 4524) were used for atrial pacing. Bipolar $45 \mathrm{~cm}(52 \mathrm{~cm}$ in the two eldest patients), $7 \mathrm{~F}$ tined ventricular leads 
(Medtronic 4024) were used for ventricular pacing in the first four patients, but owing to the problems of manipulating two leads in the subclavian veins of small children, in subsequent patients a thinner unipolar ventricular lead (Medtronic 4023) was used.

The pacemakers were implanted under general anaesthetic. The leads were introduced through the left subclavian vein. For the ventricular lead, a loop was formed in the atrium to allow for growth. The extravascular portion of each of the leads was tunnelled under the skin to the generator pocket just medial to the left axilla.

Postimplantation study follow up was for 12 months. During these 12 months the pacemaker was set to three different modes, each for a period of four months. The three different modes were: dual chamber pacing with rate drop response (DDD), ${ }^{19}$ ventricular pacing with hysteresis (VVI), and sensing only (ODO). In VVI and DDD, the pacemaker was programmed to intervene should the patient's heart rate fall below $50-55$ beats/min and the pacing intervention rate was programmed to 100-110 beats/min. The parent, patient, and doctor analysing the results were blinded to the modes.

Because the natural history of reflex anoxic seizures in most children is to improve with time, to allow for a possible temporal effect it was important to ensure that, as far as possible, there was an equal distribution of each of the pacemaker modes for each of the four month periods. This was done by allocating patients to one of six possible pacing protocols, according to chronological order of pacemaker implantation (table 1); however, only the technician and doctor programming the pacemaker were aware of the sequence of the modes in each protocol.

Before consent for the study, families were advised that the pacemaker would be set to three different modes, but that they would not be told of the setting. At the start of each mode, they were advised that the frequency of attacks might change and could even become worse. The families were given a diary to keep for each of the four month periods, in which to record and describe all attacks.
Table 1 Pacing sequences

\begin{tabular}{llll}
\hline Protocol & First 4 months & Second 4 months & Third 4 months \\
\hline 1 & DDD & VVI & ODO \\
2 & VVI & DDD & ODO \\
3 & ODO & DDD & VVI \\
4 & DDD & ODO & VVI \\
5 & VVI & ODO & DDD \\
6 & ODO & VVI & DDD
\end{tabular}

DDD, dual chamber pacing with rate drop response; ODO, sensing only, no pacing; VVI, ventricular pacing with wide hysteresis.

At the end of the study, all the diaries were analysed by a doctor blinded to the patient and pacemaker mode. The recorded events were interpreted as: full blown reflex anoxic seizure with loss of consciousness; "near miss" episode with brief cry, change in colour, but no actual loss of consciousness; and "non-specific" symptoms such as unsteadiness and dizziness.

Data were analysed considering pacemaker modes pairwise using two sided sign tests on the difference in the number of episodes between the two modes. Subjects with the same number of episodes on either mode did not contribute to that test.

The primary symptom considered was loss of consciousness. For those who had no loss of consciousness either with VVI or DDD pacing, the two pacing modes were compared for differences in the number of near miss events.

The study had approval of the local hospital ethics committee and was performed with the informed consent of the parents.

\section{Results}

Twelve patients (eight boys, four girls) were enrolled in the study (table 2). Their ages ranged from 2 years to 14.7 years (median 2.8 years, mean 4 years). Duration of recorded asystole ranged from 10 seconds to 40 seconds (mean (SD), 23 (9) seconds). Weight of patients at time of pacemaker implant ranged from 12.1 to $52.5 \mathrm{~kg}$ (mean $19.4(11.8) \mathrm{kg}$ ).

\section{COMPLICATIONS}

One patient required repositioning of an atrial lead 24 hours following implantation owing to lead displacement. One patient developed an infection at the generator site four weeks after

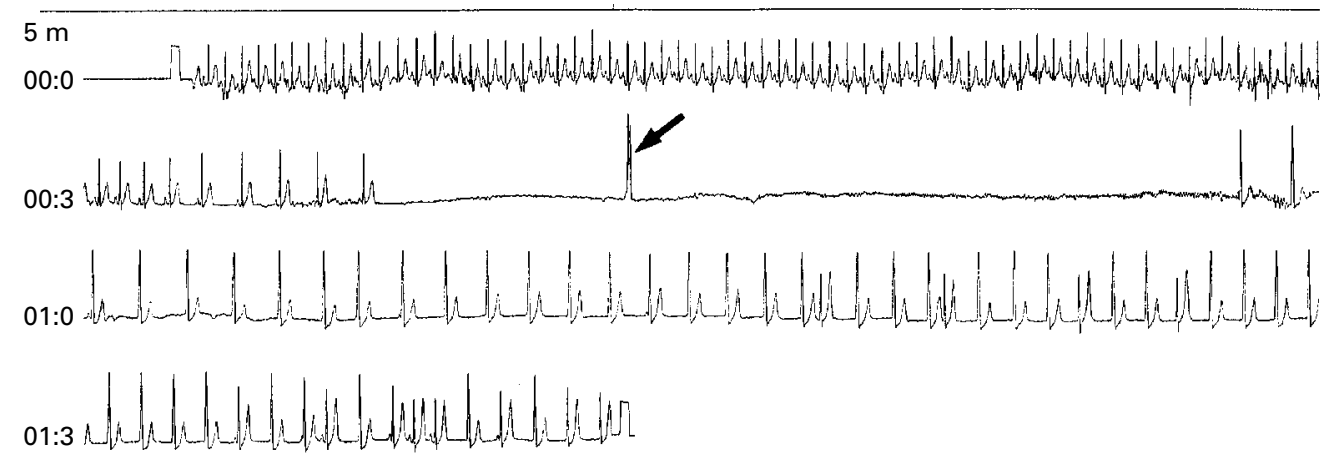

Figure 1 Episode of asystole recorded on a cardiac event monitor during a reflex anoxic seizure. The monitor was programmed to record for 40 seconds before and 60 seconds after activation of the record button (indicated by arrow). Each strip is 30 seconds. Initially there is sinus tachycardia corresponding to the child running, but in response to a noxious stimulus (in this case, a bump on the elbow) the heart slows within a few seconds to complete cardiac asystole lasting over 20 seconds. On return of cardiac rhythm there is bradycardia with a nodal rhythm of 55-60 beats/min. This continues beyond the end of the recording. 
Table 2 Frequency of symptoms following pacemaker implantation

\begin{tabular}{|c|c|c|c|c|c|c|c|c|c|c|c|c|}
\hline \multirow[b]{2}{*}{ Subject } & \multirow[b]{2}{*}{ Protocol } & \multirow[b]{2}{*}{ Age (years) } & \multirow[b]{2}{*}{ Asystole (s) } & \multicolumn{3}{|l|}{$L O C$} & \multicolumn{3}{|l|}{$N M$} & \multicolumn{3}{|l|}{ NS } \\
\hline & & & & $O D O$ & $V V I$ & $D D D$ & $O D O$ & $V V I$ & $D D D$ & $O D O$ & $V V I$ & $D D D$ \\
\hline 1 & 1 & 4.1 & 16 & - & - & - & - & - & - & - & - & \\
\hline 2 & 2 & 2.1 & 32 & 2 & 0 & 0 & 9 & 41 & 21 & 2 & 0 & 1 \\
\hline 3 & 3 & 8.5 & 22 & 0 & 0 & 0 & 0 & 0 & 0 & 57 & 54 & 27 \\
\hline 4 & 4 & 5.1 & 10 & 0 & 0 & 0 & 19 & 11 & 3 & 0 & 0 & 1 \\
\hline 5 & 5 & 3.1 & 10 & 0 & 0 & 0 & 8 & 7 & 5 & 0 & 1 & 0 \\
\hline 6 & 6 & 2.8 & 40 & 14 & 0 & 0 & 8 & 12 & 4 & 0 & 0 & 0 \\
\hline 7 & 1 & 2.6 & 19 & 3 & 0 & 0 & 14 & $\star$ & $\star$ & 0 & $\star$ & $\star$ \\
\hline 8 & 2 & 2.4 & 26 & 5 & 2 & 3 & 0 & 23 & 24 & 0 & 0 & 0 \\
\hline 9 & 3 & 2.0 & 22 & 8 & 0 & 0 & 4 & 10 & 4 & 3 & 0 & 0 \\
\hline 10 & 4 & 3.2 & 18 & 22 & 0 & 10 & 17 & 19 & 10 & 0 & 0 & 0 \\
\hline 11 & 5 & 14.7 & 28 & 4 & 0 & 0 & 1 & 1 & 0 & 0 & 13 & 0 \\
\hline 12 & 6 & 2.1 & 25 & 32 & 0 & 0 & 5 & 30 & 14 & 0 & 0 & 0 \\
\hline
\end{tabular}

* The diaries of subject 7 were lost by the parents for the periods of VVI and DDD but the parents reported that there were no episodes of loss of consciousness during either of these periods and that they preferred the four months the pacemaker was programmed to DDD as there were fewer near misses.

Subject 1 was withdrawn from the study because the pacemaker was removed due to infection.

DDD, dual chamber pacing with rate drop response; LOC, full blown reflex anoxic seizure with loss of consciousness; NM, near miss; NS, non-specific symptoms such as dizziness, unsteadiness, etc; ODO, sensing only/no pacing; VVI, ventricular pacing with hysteresis.

implantation. The system was explanted and the patient taken out of the study. A VVI pacemaker was implanted outside the study by another cardiologist 10 weeks later, but this too became infected and required explantation.

\section{RECORDED EVENTS}

Table 2 shows the frequency of symptoms following pacemaker implantation. There was no evidence of a temporal effect in terms of frequency of any symptoms. Episodes of loss of consciousness with seizures were significantly reduced both on dual chamber pacing $(p=0.0078)$ and ventricular pacing $(\mathrm{p}=0.0078)$ when compared with sensing only. There was no significant difference between ventricular pacing and dual chamber pacing in prevention of loss of consciousness, but in those patients who had no loss of consciousness with either dual chamber or single chamber pacing, there were significantly fewer near miss events with dual chamber pacing compared with ventricular pacing $(p=0.016)$. Table 3 summarises the results.

In terms of symptomatic response to pacemaker implantation, six patients had no loss of consciousness when paced compared with syncopal episodes when the pacemaker was programmed to sensing only, three patients had no further episodes of syncope whether paced or not, and two patients continued to have episodes of syncope despite being paced.

Table 3 Sign tests on pairwise comparisons between pacemaker modes

\begin{tabular}{llll}
\hline Symptom & $\begin{array}{l}\text { More episodes } \\
\text { on (a) }\end{array}$ & $\begin{array}{l}\text { More episodes } \\
\text { on (b) }\end{array}$ & $\begin{array}{l}\text { Sign test } \\
\text { p value }\end{array}$ \\
\hline $\begin{array}{l}\text { Loss of consciousness } \\
\text { (a) ODO v (b) VVI }\end{array}$ & 8 & 0 & 0.0078 \\
$\begin{array}{l}\text { (a) ODO v (b) DDD } \\
\text { (a) VVI } v \text { (b) DDD }\end{array}$ & 8 & 0 & 0.0078 \\
"Near miss"* & 0 & 2 & 0.5 \\
(a) VVI $v$ (b) DDD & 7 & 0 & 0.016 \\
\hline
\end{tabular}

Data are the number of subjects with more episodes on that pacemaker mode, with sign test $\mathrm{p}$ values.

^In patients who had no loss of consciousness during either VVI or DDD.

DDD, dual chamber pacing with rate drop response; ODO, sensing only, no pacing; VVI, ventricular pacing with wide hysteresis.
Two patients (patients 3 and 11) had unpleasant symptoms of dizziness and awareness of the pacemaker pacing when the pacemaker was set at VVI.

\section{Discussion}

This is the first blinded study demonstrating the efficacy of permanent cardiac pacing in neurally mediated syncope. The recent North American pacemaker study, in which adult patients with vasovagal syncope were randomised to either a dual chamber pacemaker or no pacemaker, showed that paced patients had a marked reduction in syncope ${ }^{2021}$ but the study did not control for the potentially powerful placebo effect of pacemaker implantation itself. $^{22}$

In our study patients acted as their own controls and we compared dual chamber and single chamber ventricular pacing in the same patient. This design helped to overcome the problem of dealing with small numbers of patients which arose as our study only accepted children with severe, frequent reflex anoxic seizures who were over 2 years old. Severe, frequent reflex anoxic seizures are relatively uncommon and mainly occur in younger children, who were excluded from our study owing to concerns about the potential problems of dual chamber implantation in very small children.

Despite the small number of patients recruited, the study showed that pacing can provide an effective treatment for patients with reflex anoxic seizures. Single chamber ventricular pacing with hysteresis was found to be as effective as dual chamber pacing with rate drop response for prevention of syncope and seizures. This is in contrast to studies in adults with vasovagal syncope where dual chamber pacing appears to be superior and ventricular pacing sometimes makes symptoms worse, possibly because of pacemaker syndrome. ${ }^{15}{ }^{16} \mathrm{It}$ may be that blood pressure in children is more dependent upon heart rate than atrioventricular synchrony, or perhaps reflex anoxic seizures represent a pure cardioinhibitory form of syncope with minimal hypotension from 
vasodilatation. It should be noted, however, that the studies in adults which have compared dual chamber with ventricular pacing for vasovagal syncope have not used high pacing intervention rates, have not been blinded, and have mainly evaluated the two modes by tilt test response rather than as crossover treatments. ${ }^{23-25}$

Although we found that ventricular pacing with hysteresis was as effective as dual chamber pacing with rate drop response for preventing syncope and seizures, the latter was more effective in reducing overall symptoms. This agrees with recent studies in adults which advocate dual chamber pacing with rate drop response as the most effective pacing programme currently available for vasovagal syncope. ${ }^{17} 18$

In terms of symptomatic response following pacemaker implantation, three patterns were identified. Six patients had no loss of consciousness when paced though they had syncopal episodes when the pacemaker was programmed to sensing only, indicating a clear benefit from pacing. Three patients had no further episodes of syncope whether paced or not, suggesting a possible placebo effect from pacemaker implantation itself. It is also possible that as reflex anoxic seizures tend to improve with age, ${ }^{26}$ these patients were improving spontaneously. Two patients continued to have episodes of syncope, albeit reduced in frequency, despite being paced. Continued episodes of syncope in spite of pacing are a finding consistent with some adult studies and a major reason why pacing for vasovagal syncope has been controversial. ${ }^{15}$ In adults, it is believed that hypotension from vasodilatation is an important factor contributing to syncope despite pacing. It may be that the two patients in our series had a significant hypotensive or even a respiratory contribution to syncope, as in so called cyanotic breath holding spells. ${ }^{27} 28$ In such patients higher pacing intervention rates might be beneficial.

Although all the patients involved in this study appeared to have some symptomatic improvement following pacemaker implantation, in future when considering children with reflex anoxic seizures for a pacemaker, it would be ideal to differentiate those who will truly benefit from pacing from those who will improve spontaneously or continue to have symptoms despite pacing. Unfortunately, the number of patients in our study was too small to identify factors which would allow this distinction to be made.

Pacemaker implantation is a significant undertaking in a small child. Owing to the small size of the patient there is an increased risk of complications at the time of implant and there are problems related to future growth. Given such problems, together with the findings of our study, we would suggest that a ventricular system with wide hysteresis is sufficient for a younger child. Older children might be more aware of the unpleasant symptoms of near misses or more troubled by pacemaker syndrome, so a dual chamber pacemaker with rate drop response may be preferable. Since the majority of toddlers will be expected to grow out of attacks, when the time comes for renewal of the pacemaker, it could be programmed to a sensing mode such as ODO to determine if episodes of syncope still occur. If not, the system can be removed and not replaced.

\section{CONCLUSIONS}

Permanent cardiac pacing provides an effective treatment for patients with severe reflex anoxic seizures. In view of the potential problems and complications of pacing in young children and the fact that most families with reflex anoxic seizures can be managed simply with explanation and reassurance, we would advise that pacing be limited to those with frequent and severe attacks in whom medical treatment has failed or is declined. Before pacemaker implantation, prolonged asystole should be demonstrated during an attack, ideally on more than one occasion. This can be done either using a cardiac event monitor or a tilt test for older children. A ventricular system is as effective as dual chamber pacing for preventing syncope and seizures, although a dual chamber pacemaker with rate drop function is more effective at reducing overall symptoms. We recommend ventricular pacing with wide hysteresis in younger children and dual chamber pacing with rate drop function in older children. Finally, we suggest that our study design could be applied to adults with vagal syncope and that an important question is not simply whether pacemakers reduce syncope but whether there are factors which identify those patients who will truly benefit from pacing from those who will continue to have attacks despite pacing and those who will improve spontaneously.

We are grateful to the parents and patients who participated in this study and for the help of Trudi Lobban of the Reflex Anoxic Seizure Information Group. We thank Professors Lawrence Weaver and Stewart Hillis for reviewing the manuscript. This study was funded by a grant from the NHS Northern Research and Development Fund.

1 DiMario FJ. Breath-holding spells in childhood. Am f Dis Child 1992;146:125-31.

2 Laxdal T, Gomez MR, Reiher J. Cyanotic and pallid syncopal attacks in children (breath-holding spells). Dev Med Child Neurol 1969;11:755-63.

3 Bridge EM, Livingston S, Tietze C. Breath-holding spells: their relationship to syncope, convulsions and other phenomena. F Pediatr 1943;23:539-61.

4 Lombroso CT, Lerman P. Breath-holding spells (cyanotic and pallid infantile syncope). Pediatrics 1967;39:563-81.

5 Stephenson JBP. Reflex anoxic seizures ("white breathholding"): non-epileptic vagal attacks. Arch Dis Child 1978; 53:193-200.

6 Stephenson JBP. Vagocardiac syncope and reflex anoxic seizures. In: Stephenson JBP, ed. Fits and faints. London:

7 Battaglia A, Guerrini R, Gastaut H. Epileptic seizures induced by syncopal attacks. $\mathcal{F}$ Epilepsy $1989 ; 2: 137-46$.

8 Stephenson JBP. Nonepileptic seizures, anoxic-epileptic seizures and epileptic-anoxic seizures. In: Wallace S, ed. Epilepsy in children. London: Chapman and Hall, 1996:526.

9 Emery ES. Status epilepticus secondary to breath-holding and pallid syncopal spells. Neurology 1990;40:859

$10 \mathrm{McW}$ illiam RC, Stephenson JBP. Atropine treatment of reflex anoxic seizures. Arch Dis Child 1984;59:473-5.

11 Palm L, Blennow G. Transdermal anticholinergic treatment of reflex anoxic seizures. Acta Paediatr Scand 1985;74:803-

12 Sapire DW, Casta A, Safley W, et al. Vasovagal syncope in children requiring pacemaker implantation. Am Heart $\mathcal{f}$ 1983;108:1406-11.

13 Narayanswami S, Whitehouse W. Permanent cardiac pacing for reflex anoxic seizures [letter]. Arch Dis Child 1996;75: 462.

14 Porter CJ, McGoon MD, Espinosa RE, et al. Apparent breath-holding spells associated with life-threatening 
bradycardia treated by permanent pacing [abstract]. Pediatr Cardiol 1994;15:260.

15 Benditt DG, Petersen M, Lurie KG, et al. Cardiac pacing for prevention of recurrent syncope. Ann Intern Med 1995;122: 204-9.

16 Sra JS, Ahktar M. Cardiac pacing during neurocardiogenic (vasovagal) syncope. I Cardiovasc Electrophysiol 1995;6 $751-60$.

17 Sheldon R, Koshman ML, Wilson W, et al. Effect of dual chamber pacing with automatic rate drop sensing on recurrent neurally mediated syncope. Am $\mathcal{F}$ Cardiol 1998;81: 158-62.

18 Benditt DG, Sutton R, Gammage MD, et al, and the International Rate-Drop Investigators Group. Clinical experience with Thera DR rate-drop response pacing algorithm in carotid sinus syndrome and vasovagal syncope. $P A C E$ 1997;20:832-9.

19 Gammage MD. Rate-drop response programming. $P A C E$ 1997;20:841-3.

20 Sheldon RS, Gent M, Roberts RS, et al, on behalf of NAVPAC. North American Vasovagal Pacemaker Study: NAVPAC. North American Vasovagal Pacemaker St
study design and organisation. PACE 1997;20:844-8.

21 Connolly SJ, Sheldon R, Roberts RS, et al. The North American Vasovagal Pacemaker Study (VPS). A rand- omized controlled trial of permanent cardiac pacing for the prevention of vasovagal syncope. $₹ \mathrm{Am}$ Coll Cardiol 99;33:16-20

2 Benditt DG. Cardiac pacing for the prevention of vasovagal syncope [editorial comment]. F Am Coll Cardiol 1999;33: 21-3.

23 Fitzpatrick A, Theodorakis G, Ahmed R, et al. Dual chamber pacing aborts vasovagal syncope induced by head-up 60 degrees tilt. PACE 1991;14:13-19.

24 Samoil D, Grubb BP, Brewster P, et al. Comparison of single and dual chamber pacing techniques in prevention of upright tilt induced vasovagal syncope. Eur 7 Cardiac Pacing Electrophysiol 1993;1:36-41.

25 Petersen MEV, Price D, Williams T, et al. Short AV interval VDD pacing does not prevent tilt induced vasovagal syncope in patients with cardioinhibitory vasovagal syndrome. PACE 1994;17:882-91.

26 Gordon N. Breath-holding spells. Dev Med Child Neurol 1987;29:805-14.

27 Samuels MP, Talbert DG, Southall DP. Cyanotic breathholding and sudden death. Arch Dis Child 1991;66:257-8.

28 Gastaut H, Gastaut Y. Electroencephalographic and clinical study of anoxic convulsions in children. Electroencephalogr Clin Neurophysiol 1958;10:607-880. 